\title{
Investigation of urinary volatile organic metabolites as potential cancer biomarkers by solid-phase microextraction in combination with gas chromatography-mass spectrometry
}

\author{
CL Silva', M Passos² and JS Câmara*,I \\ 'CQM/UMa - Centro de Química da Madeira, Centro de Ciências Exactas e da Engenharia, Universidade da Madeira, Campus Universitário da \\ Penteada, Funchal 9000-390, Portugal; ${ }^{2}$ Hospital Dr Nélio Mendonça, Avda. Luís de Camões, Funchal 9000, Portugal
}

BACKGROUND: Non-invasive diagnostic strategies aimed at identifying biomarkers of cancer are of great interest for early cancer detection. Urine is potentially a rich source of volatile organic metabolites (VOMs) that can be used as potential cancer biomarkers. Our aim was to develop a generally reliable, rapid, sensitive, and robust analytical method for screening large numbers of urine samples, resulting in a broad spectrum of native VOMs, as a tool to evaluate the potential of these metabolites in the early diagnosis of cancer.

METHODS: To investigate urinary volatile metabolites as potential cancer biomarkers, urine samples from 33 cancer patients (oncological group: 14 leukaemia, 12 colorectal and 7 lymphoma) and 21 healthy (control group, cancer-free) individuals were qualitatively and quantitatively analysed. Dynamic solid-phase microextraction in headspace mode (dHS-SPME) using a carboxenpolydimethylsiloxane (CAR/PDMS) sorbent in combination with GC-qMS-based metabolomics was applied to isolate and identify the volatile metabolites. This method provides a potential non-invasive method for early cancer diagnosis as a first approach. To fulfil this objective, three important dHS-SPME experimental parameters that influence extraction efficiency (fibre coating, extraction time and temperature of sampling) were optimised using a univariate optimisation design. The highest extraction efficiency was obtained when sampling was performed at $50^{\circ} \mathrm{C}$ for $60 \mathrm{~min}$ using samples with high ionic strengths ( $17 \%$ sodium chloride, $\mathrm{Wv}^{-1}$ ) and under agitation.

RESULTS: A total of 82 volatile metabolites belonging to distinct chemical classes were identified in the control and oncological groups. Benzene derivatives, terpenoids and phenols were the most common classes for the oncological group, whereas ketones and sulphur compounds were the main classes that were isolated from the urine headspace of healthy subjects. The results demonstrate that compound concentrations were dramatically different between cancer patients and healthy volunteers. The positive rates of 16 patients among the 82 identified were found to be statistically different $(P<0.05)$. A significant increase in the peak area of 2 -methyl3-phenyl-2-propenal, p-cymene, anisole, 4-methyl-phenol and I,2-dihydro-1,I,6-trimethyl-naphthalene in cancer patients was observed. On average, statistically significant lower abundances of dimethyl disulphide were found in cancer patients.

CONCLUSIONS: Gas chromatographic peak areas were submitted to multivariate analysis (principal component analysis and supervised linear discriminant analysis) to visualise clusters within cases and to detect the volatile metabolites that are able to differentiate cancer patients from healthy individuals. Very good discrimination within cancer groups and between cancer and control groups was achieved.

British Journal of Cancer (201 I) I 05, I894-1904. doi: I0.1038/bjc.201 I.437 www.bjcancer.com

Published online I5 November 20 II

(c) 20II Cancer Research UK

Keywords: urine; volatile organic metabolites; biomarkers; HS-SPME/GC-qMS

Cancer is characterised by abnormal growth and development of normal cells beyond their natural boundaries. Despite global efforts to limit the incidence of this disease, cancer has become the leading cause of death in the last 50 years. Specifically, breast cancer is the most common malignancy and the second most common cause of cancer-related mortality in women. Prostate cancer is the most common solid organ malignancy diagnosed in

* Correspondence: Professor JS Câmara; E-mail: jsc@uma.pt Received 20 May 201 I; revised 3 August 2011; accepted 23 September 20 I I; published online I5 November 20 I I men in Europe and the United States of America and is the second most frequent cause of cancer-related death in men (Ullah and Aatif, 2009; Jemal et al, 2010). Furthermore, after cardiovascular diseases, cancer is the second cause of death among the global population (Infante et al, 1994; Weisburger and Williams, 2000; Oliveira et al, 2007).

The factors that are responsible for cancer development are classified as exogenous and endogenous (Oliveira et al, 2007). The first group includes nutritional habits (food preservation and preparation), socio-economic status, lifestyle, physical agents (ionising and non-ionising radiation), chemical compounds (natural and synthetic) and biological agents (Helicobacter pylori, 
Epstein-Barr virus, human $\mathrm{T}$ lymphotropic viruses $\mathrm{I}$ and II, human papilloma virus and the hepatitis B virus, as well as parasites, such as Schistosoma haemotobium, Clonorchis sinensis and Opisthorchis vivarium) (Minamoto et al, 2000; Weisburger and Williams, 2000). Unhealthy lifestyle habits such as excess alcohol consumption, the inhalation of tobacco and related products, the ingestion of certain foods and their contamination by mycotoxins are responsible for higher incidences of certain types of neoplasias in a number of population groups (Weisburger and Williams, 2000; Oliveira et al, 2007). Endogenous factors include immune system damage and inflammation caused by uncertain aetiology (e.g., ulcerative colitis and pancreatitis), genetic makeup, age, endocrine balance and physiological condition (Barrett and Anderson, 1993; Infante et al, 1994).

The management of high-risk cancers requires diagnosis at an early stage, which specifies the need for specific and sensitive biomarkers. Sometimes, certain molecules are differentially expressed in cancer cells relative to their normal counterparts, and their altered levels can be measured to establish a correlation with the diseased state (Cassiday, 2006; Jemal et al, 2010). With the development of high-throughput techniques for biomarker discovery, the field of cancer biomarkers has rapidly expanded (Lauridsen et al, 2007; Gramolini et al, 2008; Gaspar and Lopes, 2009; Jiang and Ma, 2009; Matsumura et al, 2010). Current biomarker candidates from blood, sputum and urine include many classes of molecules, such as proteins, tumour-specific antigens, anti-tumour antibodies, cell type-specific peptides, various metabolic products and epigenetic phenomena (e.g., hyper-methylated DNA, RNA and specific gene expression) (Greenberg and Lee, 2007; Matsumura et al, 2010); however, according to Matsumura et al (2010), no biomarker identified to date has been shown to have adequate sensitivity, specificity and reproducibility so as to be considered sufficient for use in the detection and monitoring of lung cancer development.

One promising class of biomarkers for cancer could be lowmolecular-weight volatile organic metabolites (VOMs) (Matsumura et al, 2010). The 'volatile hypothesis', which was initially postulated for lung cancer, has led to a number of studies examining the utility of analysing these metabolites in the exhaled breath of animals (such as dogs) or in sophisticated biochemical techniques (McCulloch et al, 2006; Belda-Iniesta et al, 2007; Song et al, 2010). Thousands of VOMs are present in trace amounts in human breath, and different studies have shown that VOM profiles of patients with lung cancer can be discriminated from those of healthy subjects (Phillips et al, 2003, 2006; Mazzone, 2008; Yung, 2010). A recent study from the Chen group using solid-phase micro-extraction (SPME) followed by gas chromatography (GC) has demonstrated that 1-butanol and 3-hydroxy-2-butanone were found at significantly higher concentrations in the breath of lung cancer patients in comparison with the controls (Chen et al, 2007).

To date, current screening trials have primarily focused on imaging modalities, such as computer tomography, magnetic resonance imaging, endoscopy and ultrasonography, together with clinical analysis; however, these methods are time-consuming and unpleasant for patients and require skilled medical staff and expensive devices. Over the past few years, however, non-imaging methods have been investigated.

Urine and breath analysis for the routine monitoring of metabolic disorders has attracted a considerable amount of scientific interest, especially because their sampling is non-invasive. These types of analyses are totally painless and agreeable to patients and can be performed as often as needed.

Apart from providing the possibility of non-invasive diagnostic testing, urine has other advantages over breath, serum or cerebrospinal fluid (Deng et al, 2004b; Xue et al, 2008). Most important, many metabolites occur in urine at nearly the same concentrations as in the plasma, whereas the total volatile metabolites in the plasma are relatively low $\left(\mu \mathrm{g}-\mathrm{ngl}^{-1}\right)$. Thus, the relative enrichment of volatile components makes urine an attractive target for a volatile metabolomic profiling approach. Urinary metabolomic studies have already been applied to the breast (Chen et al, 2009; Henneges et al, 2009), lung (Carrola et al, 2011), prostate (Chikkaveeraiah et al, 2009; Sreekumar et al, 2009; Jentzmik et al, 2010), colorectal (Ma et al, 2009; Qiu et al, 2010) and liver cancers (Chen et al, 2009).

In recent years, there has been an enormous effort to develop specific and sensitive biomarkers for the precise and accurate screening, diagnosis, prognosis and monitoring of high-risk cancer to assist with therapeutic decisions. Different methods have thus been developed to analyse volatile metabolites and compare them in healthy subjects and cancer patients, such as chemical interaction, adsorptive binding, cold trapping and supercritical fluid extraction. The most successful methods in this field are SPME and the recently developed multi-bed sorption trap (Giardina and Olesik, 2003; Prado et al, 2003; Yu et al, 2009). This technique was developed by Pawliszyn in late 1989 as a new pre-concentration technology in which a fused coated silica fibre is used as the stationary phase (Ouyang et al, 2005; Song et al, 2010). This methodology presents several advantages over conventional solvent extraction procedures: SPME is rapid, easy to use, solvent free and sensitive, and does not require any concentration step before analysis, preventing artefacts (Musteata and Pawliszyn, 2007).

Therefore, in this study, we tested the potentialities of a simple and solvent-free miniaturised high-throughput analytical methodology without sample derivatisation based on dynamic solidphase microextraction in headspace mode (dHS-SPME) in combination with GC-qMS. This technique was used for the metabolomic analysis of urine samples that were obtained from clinically diagnosed patients with leukaemia, colorectal and lymphoma cancers, and from healthy controls (cancer-free) to provide comprehensive information on volatile metabolites as potential cancer biomarkers.

A comparative analysis of the urine metabolome between cancer patients and normal controls was carried out. This study presents some preliminary results in a small group of cancer patients in comparison with the controls. Six different stationary phases, polydimethylsiloxane (PDMS), polyacrylate (PA), divinylbenzene/ carboxen/PDMS (DVB/CAR/PDMS), carbowax/DVB (CW/DVB), $\mathrm{CAR} / \mathrm{PDMS}$ and $\mathrm{PDMS} / \mathrm{DVB}$, providing specificity for a wide range of polar and non-polar volatile compounds, were compared in terms of extraction efficiency and sensitivity. Factors that might affect the dHS-SPME procedure, such as extraction time and extraction temperature, were also investigated to determine the analytical performance of the selected fibre.

To test the applicability of our method, a total of 54 urine samples (healthy individuals, $n=21$ and cancer patients, $n=33$ ) were collected in the Hospital Dr Nélio Mendonça (HaematoOncology Unit) and analysed. Multivariate statistical methods were used to gain insight into the metabolomic differences between healthy and patient cases and find related volatile metabolites that could be associated with a unique type of cancer (e.g., leukaemia, colorectal, lymphoma). This identification is indispensable for future work on the biochemical sources of these compounds and their metabolic pathways.

\section{MATERIALS AND METHODS}

\section{Reagents and materials}

Sodium chloride $(\mathrm{NaCl})$ and hydrogen chloride $(\mathrm{HCl})$ were purchased from Panreac (Barcelona, Spain) and Sigma-Aldrich (St Louis, MO, USA), respectively. Ultra-pure water from a Milli-Q system (Millipore, Bedford, MA, USA) with a conductivity of 
$18 \mathrm{M} \Omega$ was used throughout. Helium at a purity of $99.999 \%$ (Air Liquide, Lisbon, Portugal) was utilised as the GC carrier gas. A Cimarec digital stirring hot plate was supplied by Thermo Scientific (Waltham, MA, USA). The SPME holder for manual sampling of SPME fibres and glass vials was purchased from Supelco (Bellefonte, PA, USA). The SPME fibres were conditioned as recommended by the manufacturer, but below the maximum recommended temperature before their first use. Before the first daily analysis, the fibres were conditioned for $5 \mathrm{~min}$ at the operating temperature of the GC injector port and the blank level was checked. The analyses were performed in triplicate.

\section{Subjects and sample collection}

The subjects were divided into four groups: normal controls and cancer patients with leukaemia, colorectal and lymphoma (Table 1). Normal controls $(n=21$, age $=44.2 \pm 10.3($ range $28-60)$ years, 18 male and 3 female) volunteered and were eligible to participate in the study if they were 18 years of age or older and had no previously diagnosed cancer.

The subjects were selected from among the blood donors at the Hospital Dr Nélio Mendonça (Funchal, Portugal). Urine samples were collected at the Blood Bank of Funchal Hospital. In all, 33 urine specimens from patients with different cancer pathologies (oncological group) were collected at the Unit of HaematologyOncology at the same institution. Leukaemia $(n=14$ age $=50.1 \pm 12.4(40-74)$ years, 6 male and 8 female), colorectal cancer $(n=11$, age $=62.0 \pm 11.7(49-78)$ years, 8 male and 3 female) and lymphoma $(n=7$, age $=42.0 \pm 19.1(18-68)$ years, 6 male and 1 female) patients were identified using specific examinations. As can be observed from these data, the groups were small and not balanced. Each individual (either patient or healthy volunteer) provided a sample of morning urine (after overnight fasting) in a $20 \mathrm{ml}$ sterile PVC container. The samples were frozen at $-80^{\circ} \mathrm{C}$ and retained until needed for the experiments. Before extraction, the $\mathrm{pH}$ values of the $25 \mathrm{ml}$ urine samples were adjusted to $1-2$.

All subjects signed an informed consent to participate in the study, and the research was approved by the Ethics Committee of Funchal Hospital.

\section{dHS-SPME procedure}

The development of a suitable dHS-SPME method to establish a urinary volatile metabolomic profile from cancer patients and healthy volunteers involved the selection and optimisation of multiple parameters that influenced extraction. In particular, the nature of the adsorptive phase, the sampling temperature and the extraction time required to achieve equilibration between the analytes and the fibre were considered by applying a univariate experimental design.

Fibre selection was performed by testing and comparing the extraction efficiency of six SPME fibres (Supelco) to different stationary phases and various film thicknesses, including PDMS

Table I The characteristics of subjects

\begin{tabular}{lcccc}
\hline & \multicolumn{3}{c}{ Patients } & \\
\cline { 2 - 4 } & Colon & Leukaemia & Lymphoma & $\begin{array}{c}\text { Normal } \\
\text { controls }\end{array}$ \\
\hline Number & $1 \mid$ & 14 & 7 & 21 \\
Mean age (s.d.) & $62.0(1 \mid .7)$ & $50.1(12.4)$ & $42.0(19.1)$ & $44.1(10.3)$ \\
Gender (male/female) & $8 / 3$ & $6 / 8$ & $6 / 1$ & $18 / 3$ \\
Current smokers & 0 & 0 & 2 & 3 \\
Ex-smokers & 0 & 0 & 1 & 0 \\
\hline
\end{tabular}

Abbreviation: s.d. $=$ standard deviation.
(100 $\mu \mathrm{m})$, PA $(85 \mu \mathrm{m})$, DVB/CAR/PDMS $(50 / 30 \mu \mathrm{m})$, CAR/PDMS $(75 \mu \mathrm{m}), \mathrm{CW} / \mathrm{DVB}(70 \mu \mathrm{m})$ and PDMS/DVB $(65 \mu \mathrm{m})$. A urine sample from a normal subject was used as the matrix for the optimisation of the dHS-SPME parameters. Frozen urine samples were completely thawed at room temperature before use.

For dHS-SPME optimisation, $4 \mathrm{ml}$ aliquots of urine samples that had been adjusted to a $\mathrm{pH}$ of $1-2$ with $50 \mathrm{ml}$ of $5.0 \mathrm{M} \mathrm{HCl}$ were placed in an 8-ml headspace glass vial. After the addition of $0.8 \mathrm{~g}$ of $\mathrm{NaCl}$ and stirring $(0.5 \times 0.1-\mathrm{mm}$ bar $)$ at 800 r.p.m., the vial was capped with a Teflon (PTFE) septum and an aluminium cap (Chromacol, Hertfordshire, UK).

The addition of salt greatly increased the extraction efficiency for many of the metabolites, particularly the polar ones. The presence of salt can influence adsorption in two ways: by changing the properties of the phase boundary and decreasing the solubility of hydrophilic metabolites in the aqueous phase (salting-out effect). This salting-out effect is widely used to increase the sensitivities of analytical methodologies.

The sample vial was placed in a thermostat bath that was adjusted to $50.0 \pm 0.1^{\circ} \mathrm{C}$ and then the SPME fibre was inserted into the headspace for $60 \mathrm{~min}$. After sampling, the SPME fibre was withdrawn into the needle, removed from the vial and inserted into the injector port $\left(250^{\circ} \mathrm{C}\right)$ of the GC-qMS system for $6 \mathrm{~min}$, wherein the metabolites were thermally desorbed and transferred directly to the analytical column. Each sample was analysed in triplicate. Blanks, which corresponded to analyses of coating fibres that were not submitted to any extraction procedure, were run between sets of three analyses. A CAR/PDMS fibre was used to investigate the extraction temperature and time. Extraction was performed at temperatures of $30^{\circ} \mathrm{C}, 50^{\circ} \mathrm{C}$ and $60^{\circ} \mathrm{C}$ for $30,45,60$ and 75 min with a stirring rate of 800 r.p.m. The optimum conditions were determined by the sum of the peak areas obtained under each parameter, number of extracted metabolites and reproducibility.

\section{GC-qMS analysis}

The SPME fibre with absorbed/adsorbed (depended on the fibre coating) volatile metabolites was inserted into the injection port of an Agilent Technologies 6890N Network gas chromatograph system (Palo Alto, CA, USA), and metabolites were desorbed for $6 \mathrm{~min}$ at $250^{\circ} \mathrm{C}$. The gas chromatograph was equipped with a $30 \mathrm{~m} \times 0.25 \mathrm{~mm}$ ID $\times 0.25 \mu \mathrm{m}$ film thickness BP-20 (SGE, Dortmund, Germany) fused silica capillary column and interfaced with an Agilent 5975 quadrupole inert mass selective detector. We employed the following chromatographic protocol for separation before the MS analyses: $35^{\circ} \mathrm{C}$ for $2 \mathrm{~min}$, and then programmed at $2.5^{\circ} \mathrm{C} \mathrm{min}^{-1}$ to $220^{\circ} \mathrm{C}$ with a 5 -min hold at this final temperature, for a total GC run time of $77 \mathrm{~min}$. The column flow was maintained constant at $1 \mathrm{ml} \mathrm{min}^{-1}$ using He (Helium N60, Air Liquide) as the carrier gas. The injection port was operated in the splitless mode and held at $250^{\circ} \mathrm{C}$.

For the 5975MS system, the operating temperatures of the transfer line, quadrupole and ionisation source were 270, 150 and $230^{\circ} \mathrm{C}$, respectively. Electron impact mass spectra were recorded at a $70 \mathrm{eV}$ ionisation voltage and the ionisation current was $10 \mu \mathrm{A}$. The acquisitions were performed in Scan mode $(30-300 \mathrm{~m} / \mathrm{z})$, and the electron multiplier was set to the autotune procedure. Metabolite identification was accomplished through manual interpretation of spectra and matching against the Agilent MS ChemStation Software (Palo Alto, CA, USA) or commercially available standard samples, when available. This software was equipped with an NIST05 mass spectral library with a similarity threshold of $>80 \%$. A series of $\mathrm{C}_{8}-\mathrm{C}_{20} n$-alkanes were analysed using the same methodology (dHS-SPME $\mathrm{CAR}_{\mathrm{PDMS}} / \mathrm{GC}-\mathrm{qMS}$ ) to establish the retention indices and to confirm the identity of the metabolites via comparison with the literature. 


\section{Statistical analysis}

Data statistical analysis was performed using the SPSS 17.0 package for Windows (SPSS Inc., Chicago, IL, USA). Significant differences among the groups were assessed with a one-way analysis of variance (ANOVA). The least square difference (LSD) test $(P$-value $<0.05)$ was used to compare the means. Principal component analysis (PCA) was also applied to the analysed groups to verify the distribution of the variables for the referred groups.

\section{RESULTS AND DISCUSSION}

The sequence followed in this study consisted of two steps. The first focused on obtaining the best experimental conditions to extract volatile metabolites from the urine of healthy and cancer volunteers using dHS-SPME. In the second step, an objective comparison of the metabolomic patterns found in the urine from cancer patients and healthy volunteers was established in terms of qualitative (identification by comparing the MS spectrum with the kóvats index) and semiquantitative (peak area ratio) differences under optimised conditions. Different profiles for healthy people and cancer patients were successfully recognised. Among other compound classes, aldehydes, ketones, terpenoids, acids, furans, volatile phenols, benzene derivatives, sulphur-containing compounds and naphthalenes were identified.

\section{Optimisation of the dHS-SPME parameters}

The optimisation of the different parameters involved in HS-SPME was performed by choosing the conditions that achieved the maximum response in terms of metabolite peak area, number of detected metabolites and reproducibility.

\section{Fibre selection}

As far as selection of the SPME fibre was concerned, the nature of the metabolites influenced which SPME fibre was chosen. Therefore, six SPME fibres were tested to select the most appropriate to isolate volatile metabolites from urine. The results of the relative extraction efficiency for the tested fibres are shown in Figure 1A.

By comparing all of the tested fibres in terms of chromatographic areas, the number of identified metabolites and relative standard deviation (r.s.d.), the best efficiency was obtained using a CAR/PDMS coating, whereas the lowest efficiency was obtained using the PDMS and PA fibres. Each extraction was performed in triplicate and the repeatability (\% r.s.d.) was lower than $20 \%$. Thus, the CAR/PDMS fibre (Figure 1A) was chosen as the SPME fibre for the remaining optimisation studies.

\section{Extraction temperature and time}

Using an extraction time of $60 \mathrm{~min}$ with $4 \mathrm{ml}$ of the same urine sample, the effect of extraction temperature at 30,50 and $60^{\circ} \mathrm{C}$ on SPME extraction efficiency was investigated. The volatile metabolites were extracted under acidic ( $\mathrm{pH} 1-2)$ conditions using only the CAR/PDMS fibre. The plot of the peak areas $v s$ extraction temperatures is shown in Figure 1B.

Temperature will substantially affect the diffusion rates of VOMs. Raising the temperature progressively from 30 to $50^{\circ} \mathrm{C}$ increased the number of extracted metabolites that were identified. Although there was a slight increase in the number of metabolites that were identified at $60^{\circ} \mathrm{C}(2$ more $)$, the r.s.d. obtained therein was higher than those for the other investigated temperatures. At high temperatures (above $50^{\circ} \mathrm{C}$ ), there is a probable degradation of the sample; hence, $50^{\circ} \mathrm{C}$ was used for the remainder of the study. As outlined in Figure $1 \mathrm{~B}$, the temperature was fixed at $50^{\circ} \mathrm{C}$ for the

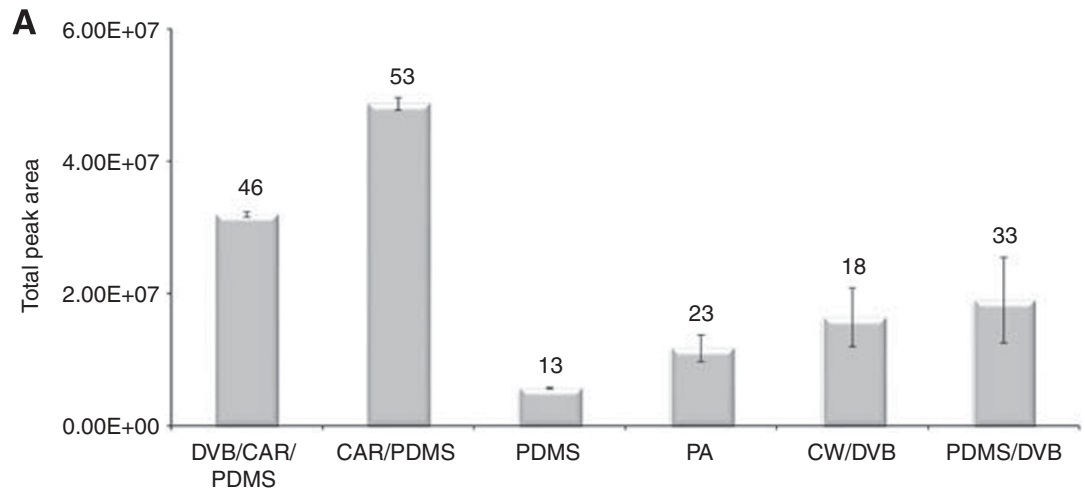

B

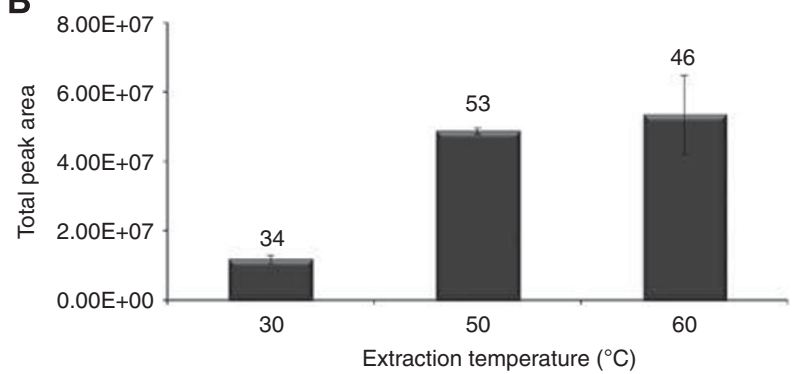

C

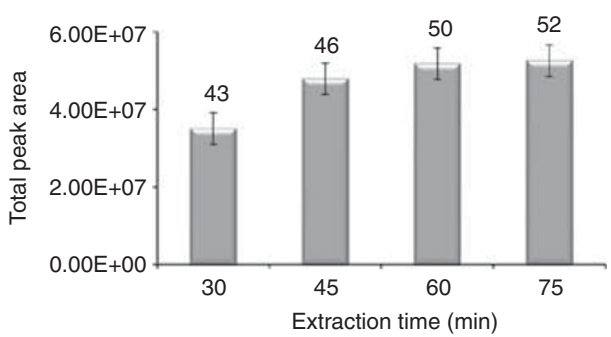

Figure I Optimisation of the solid-phase micro-extraction (SPME) influencing extraction parameters: (A) effect of fibre coatings (60 min of extraction time at $50^{\circ} \mathrm{C}$ ); (B) effect of extraction temperature (fibre: $75 \mu \mathrm{m}$ carboxen-polydimethylsiloxane (CAR/PDMS); extraction time: 60 min); and (C) influence of the extraction time (fibre: $75 \mu \mathrm{m}$ CAR/PDMS; extraction temperature: $50^{\circ} \mathrm{C}$ ) on SPME extraction efficiency of urinary volatile metabolites in a healthy individual. All assays were carried out with continuous stirring (800 r.p.m.). Thermal desorption of metabolites were performed at $250^{\circ} \mathrm{C}$ for 6 min. Numbers above the bars represent the total number of metabolites identified. 


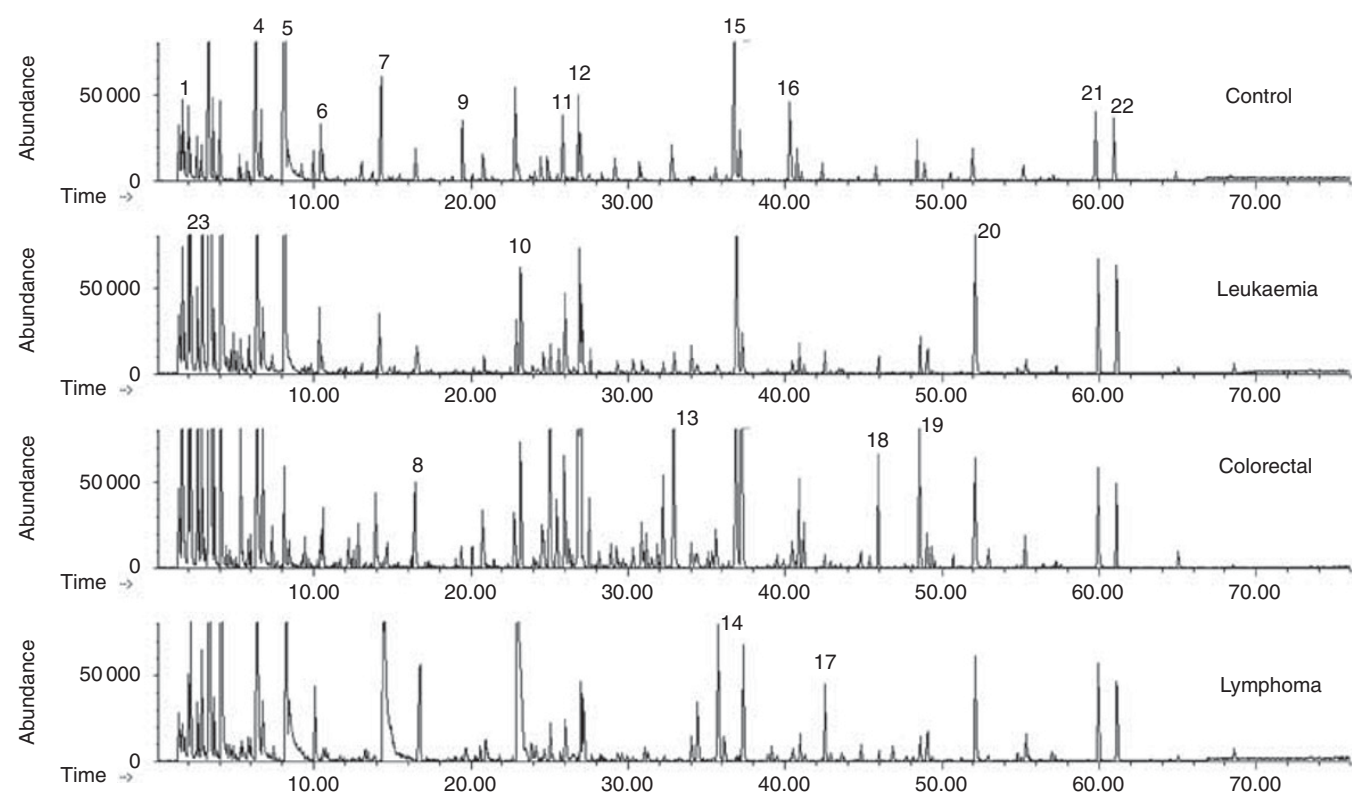

Figure 2 A typical urinary gas chromatograph quadrupole mass spectrometer (GC-qMS)-based metabolomics profile (fingerprint signals) of cancer patients (leukaemia, colorectal and lymphoma) contrasted with a healthy volunteer (control group). Extraction was performed using a carboxenpolydimethylsiloxane (CAR/PDMS) coating exposed to the headspace vapour for $60 \mathrm{~min}$ at $50^{\circ} \mathrm{C}$. Peak identification: (I) methanethiol; (2) acetone; (3) 2-pentanone; (4) dimethyl disulphide; (5) 4-heptanone; (6) 4-methyl-2-pentanol (IS); (7) $\gamma$-terpinene; (8) 2-methoxythiophene; (9) dimethyl trisulphide; ( I0) linalool oxide; (I I) 2-ethyl-I-hexanol; (I2) vitispirane I; (I3) menthol; (I4) 4,7-dimethyl-benzofuran; (I5) I,2-dihydro- I,I,6-trimethyl-naphthalene; ( 16$) \beta$-damascenone; ( 7 ) p-cimenol; ( 18) 3,4-dimethyl-benzaldehyde; (I9) I-ethyl-3,5-diisopropyl-benzene; (20) 4-methyl-phenol; (2I) p-tert-butyl-phenol; and (22) 2,4-bis(I,I-dimethylethyl)-phenol.

A

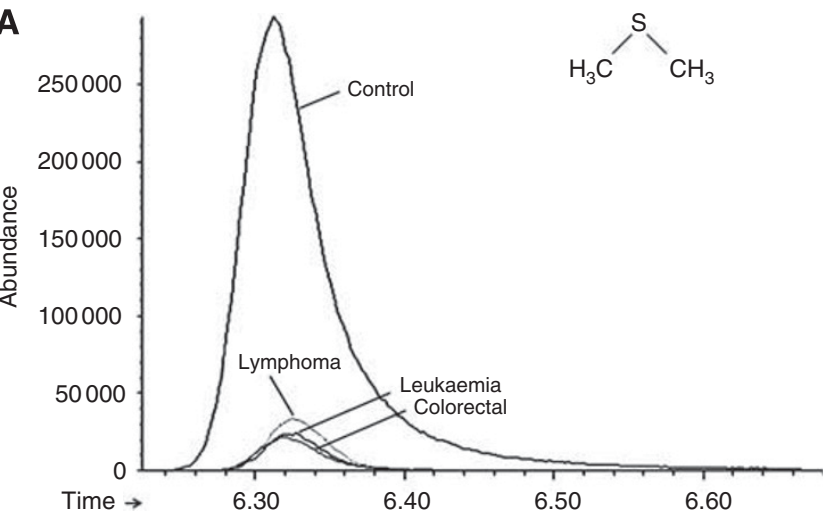

C

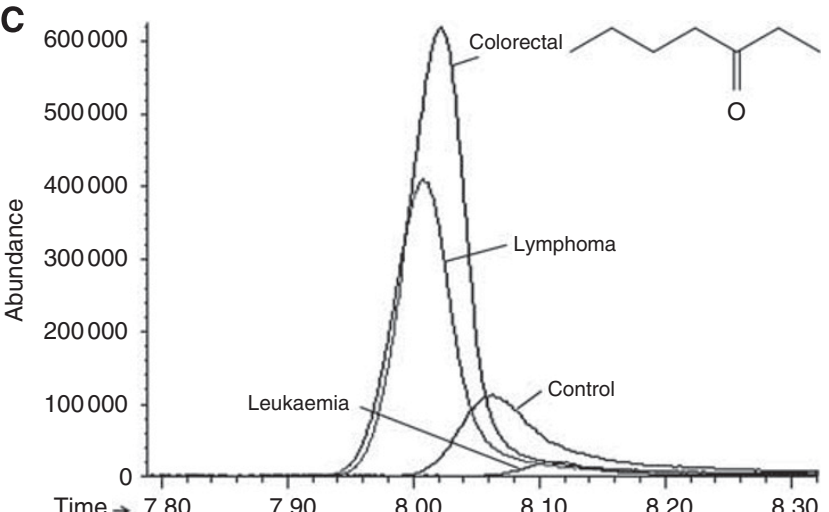

$B_{140000}^{B}$

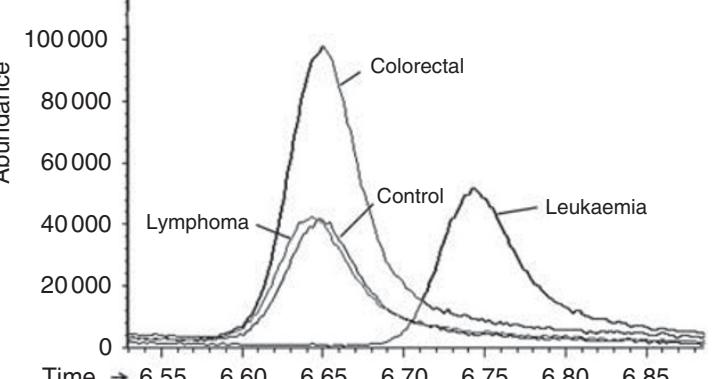

D

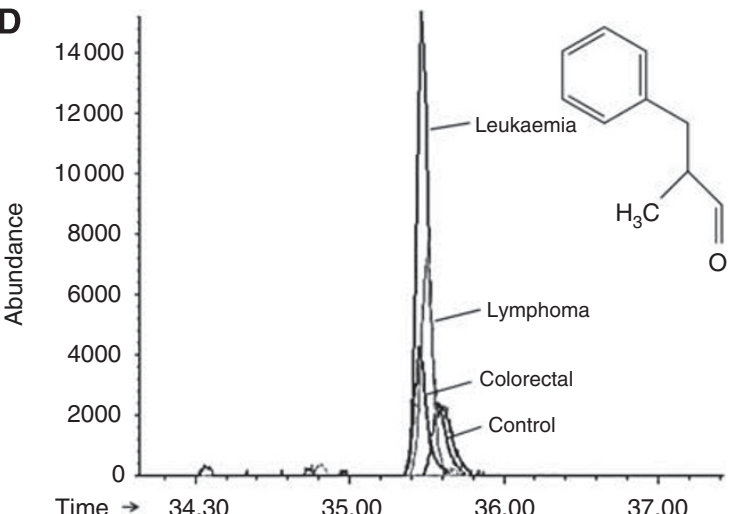

Figure 3 Comparison between healthy and oncological group of four illustrative metabolites selected from 82 peaks analysed. Enlarged part of the chromatograms of Figure 2 with peaks representing: (A) dimethyl disulphide (ion 94); (B) hexanal (ion 44); (C) 3-heptanone (ion 57); and (D) 2-methyl-3phenyl-2-propenal (ion 145). 
extraction of urinary volatile metabolites from healthy volunteers and cancer patients.

The influence of extraction time on the efficiency of the SPME process was investigated by exposing the SPME fibre to the urine headspace at $50^{\circ} \mathrm{C}$ for $30,45,60$ and $75 \mathrm{~min}$. Sorption time profiles for volatile metabolites indicated that a sampling time of $>45 \mathrm{~min}$ was necessary to reach equilibrium. When the equilibrium was not reached, an alternative methodology was to develop the extraction under non-equilibrium conditions, which require shorter extraction times. Figure $1 \mathrm{C}$ demonstrates that the equilibrium between the samples and fibre was established in $60 \mathrm{~min}$. With additional extraction time, there was no obvious increase in the peak area. On the basis of the results, $60 \mathrm{~min}$ was chosen as the extraction time for further analysis.

\section{Characterisation and comparative analysis of urinary volatile metabolites}

We next characterised the nature of the chemical variation in the collected urine samples to distinguish individuals with tumours from those without by analysing urinary volatile metabolites using solid-phase microextraction in combination with GC coupled to mass spectrometry. According to the typical GC-qMS total ion chromatograms (TICs) depicted in Figure 2, a large and diverse set of metabolites can be distinguished in the urine obtained from healthy people (control group) and that obtained from cancer patients (leukaemia, colorectal and lymphoma).

Different urinary GC-qMS profiles for healthy people and cancer patients were able to be recognised. In all, 82 of the identified volatile metabolites that were found in both the cancer and the healthy urine samples included a variety of chemical structures and were identified to be involved in multiple biological functions (e.g., pheromonal communication for 2-heptanone; Deng et al (2004a)). Some metabolites that have previously been reported to appear in human urine (dimethyl disulphide, methanethiol and 2-methylbutanoic acid) (Henneges et al, 2009) were also identified. The enlarged peaks in the TICs of some of the significant metabolites are depicted in Figure 3, and these facilitate the differentiation of metabolomic profiles.

The peak area ranges (minimum, maximum and median values) of the urinary volatile metabolites that were found in the cancer patients and healthy subjects are summarised in Supplementary Table 1. Identification was performed using a NIST05 library through comparison of fragmentation patterns with a standard mass chromatogram and verified by reference compounds, where available.

The metabolomic origin and physiological function of most of the VOMs are still not known. Their origins lie in a variety of endogenous biochemical pathways and exogenous sources (environmental, unhealthy lifestyle habits, biological agents); however, the chemical pathways of generation have not yet been explained. Some of the endogenous markers were derived from the mevalonic acid pathway of cholesterol synthesis (e.g., unsaturated hydrocarbons like isoprene), from glucose metabolism (e.g., acetone) and from the oxygen free radical-mediated lipid peroxidation of fatty acids (e.g., aldehydes and linear and branched saturated hydrocarbons). The source of the VOMs that was identified to derive from naphthalene is not yet known; they may be the degradation products of steroids. Further research would be required to determine which of these metabolites are of tumour origin and which originate from normal metabolic processes, as well as which are down- or upregulated by tumour growth.

Variation in the peak areas of identified metabolites clearly showed differences in the relative amounts of various metabolites for different individuals (Supplementary Table 1 and Figure $4 \mathrm{~A}$ and $\mathrm{B})$. The identified metabolites belonged to several distinct chemical families: aldehydes, ketones, terpenoids, acids, alcohols, benzene derivatives, furan and sulphur-containing compounds,
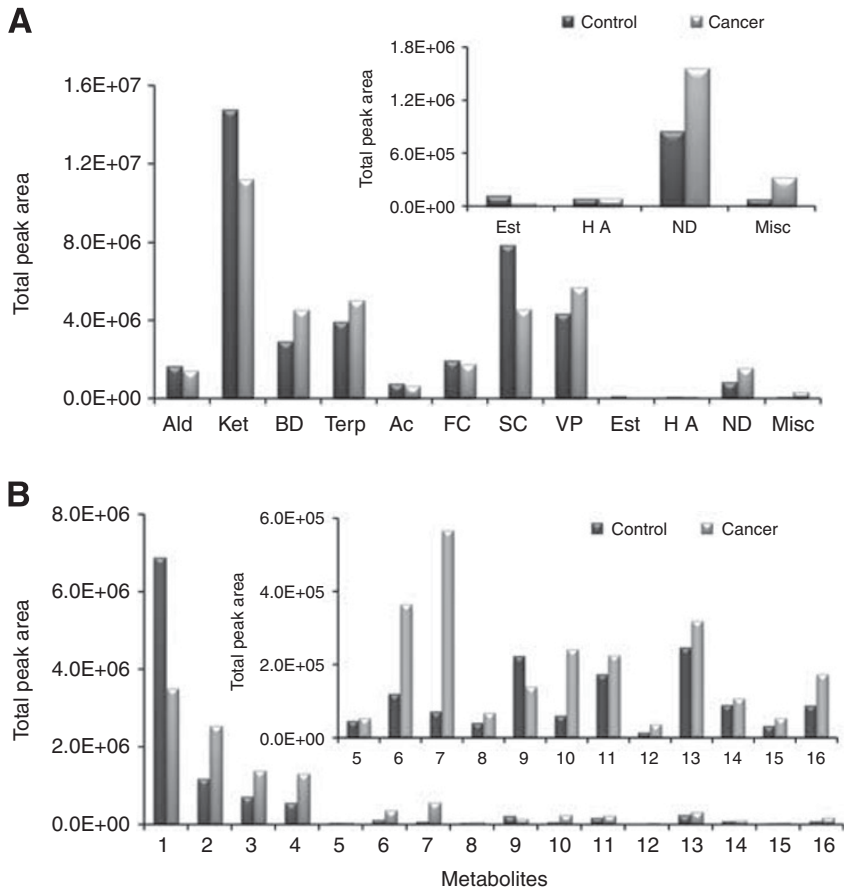

Figure 4 Average levels of metabolites excreted in urine samples from normal subjects (2I) and cancer patients (3I). (A) Chemical families identified in oncological and control groups (Ald - aldehydes; Ket ketones; BD - benzene derivates; Terp - terpenoids; Ac - acids; FC - furan compounds; SC - sulphur compounds; VP - volatile phenols; Est - esters; HA - higher alcohols; ND - naphthalene derivates; Misc - miscellaneous). (B) Average areas for statistically significant metabolites identified in cancer patients. Numbered bars correspond to: (I) hexanal; (2) heptanal; (3) 2 methyl-3-phenyl-2-propenal; (4) 3-heptanone; (5) 1,2,4-trimethylbenzene; (6) p-cymene; (7) anisole; (8) $\gamma$-terpinene; (9) bornylene; (10) dimethyl disulphide; (I I) 2-methoxythiophene; (12) 4-methyl-phenol; (13) I-octanol; (14) I,2-dihydro-I, I,6-trimethyl-naphthalene; (I5) I,4,5-trimethyl-naphthalene; and (I6) 2,7-dimethyl-quinoline.

phenols, esters, naphthalene derivatives and miscellaneous (Figure 4A).

We observed relatively consistent changes for many metabolites in samples obtained from the cancer group, with the most common pattern being decreased production (downregulation) in the cancer group samples and either increased production (upregulation) or a negligible change in the control group. For example, volatile sulphur-containing metabolites such as dimethyl disulphide, methanethiol, dimethyl trisulphide and methoxythiophene, which are generated in humans by the incomplete metabolism of methionine in the transamination pathway, were dramatically downregulated as a consequence of neoplastic cell presence (Supplementary Table 1). Thus, an overall downregulation of these metabolites may be a common feature of tumour growth.

For the control group, the largest chemical classes in the metabolomic profile were ketones, sulphur compounds, volatile phenols and terpenoids. The major metabolites of these chemical families are 4-heptanone, dimethyl disulphide, $p$-tert-butyl phenol and D-carvone, respectively. The origin of 4 -heptanone is still unknown, but it is probably from an exogenous source (Deng et al, $2004 \mathrm{~b})$. It has previously been reported that 4-heptanone is produced from the in vivo metabolism of plasticisers in humans (Walker and Mills, 2001). Esters and higher alcohols were the chemical classes with the smallest contributions to the volatile metabolomic profile.

Ketones, volatile phenols, terpenoids and benzene derivatives were the chemical groups that contributed most to the 


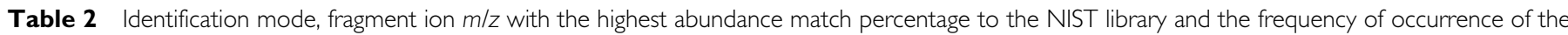
identified metabolites in patients and normal controls

\begin{tabular}{|c|c|c|c|c|c|c|c|}
\hline \multirow[b]{2}{*}{ Metabolites } & \multirow[b]{2}{*}{ ID $^{\mathbf{a}}$} & \multirow[b]{2}{*}{$\mathrm{m} / \mathbf{z}$} & \multirow[b]{2}{*}{ Match per cent } & \multicolumn{4}{|c|}{ Frequency of occurrence (\%) } \\
\hline & & & & Colorectal & Leukaemia & Lymphoma & Control \\
\hline Methanethiol $^{b}$ & MS & 47 & 80 & $100.0^{c}$ & 100.0 & 100.0 & 100.0 \\
\hline Furan $^{\text {b }}$ & St, MS & 68 & 91 & 100.0 & 100.0 & 100.0 & 100.0 \\
\hline Acetone $^{\mathrm{b}}$ & St, MS & 43 & 80 & 100.0 & 100.0 & 100.0 & 100.0 \\
\hline 2-Methylfuran ${ }^{\text {b }}$ & MS & 82 & 87 & 100.0 & 100.0 & 100.0 & 100.0 \\
\hline Ethyl acetate & St, MS & 43 & 85 & 33.3 & 71.4 & 71.4 & 71.4 \\
\hline 2-Butanone ${ }^{b}$ & St, MS & 43 & 82 & 100.0 & 100.0 & 100.0 & 100.0 \\
\hline 2-Methyl-butanal & MS & 57 & 87 & 33.3 & 42.9 & 57.1 & 71.4 \\
\hline 3-Methyl-butanal & St, MS & 44 & 81 & 33.3 & 7.1 & 14.3 & 28.6 \\
\hline 2,5-Dimethyl-furan & MS & 96 & 85 & 91.7 & 100.0 & 100.0 & 100.0 \\
\hline 2-Pentanone ${ }^{\mathrm{b}}$ & St, MS & 43 & 86 & 100.0 & 100.0 & 100.0 & 100.0 \\
\hline Methyl isobutyl ketone & St, MS & 43 & 93 & 41.7 & 71.4 & 71.4 & 100.0 \\
\hline Toluene & St, MS & 91 & 86 & 91.7 & 100.0 & 85.7 & 100.0 \\
\hline I-(2-furanyl)ethanone ${ }^{\mathrm{b}}$ & St, MS & 95 & 80 & 100.0 & 100.0 & 100.0 & 100.0 \\
\hline Dimethyl disulphide ${ }^{\mathrm{b}}$ & St, MS & 94 & 97 & 100.0 & 100.0 & 100.0 & 100.0 \\
\hline 3-Hexanone & St, MS & 43 & 84 & 100.0 & 100.0 & 100.0 & 100.0 \\
\hline Hexanal $^{b}$ & St, MS & 44 & 90 & 100.0 & 100.0 & 100.0 & 100.0 \\
\hline Geraniol oxide & MS & 139 & 90 & 41.7 & 57.1 & 57.1 & 90.5 \\
\hline 4-Heptanone & St, MS & 71 & 91 & 100.0 & 100.0 & 100.0 & 100.0 \\
\hline p-Xylene & MS & 91 & 88 & 16.7 & 21.4 & 42.9 & NF \\
\hline 3-Heptanone & St, MS & 57 & 89 & NF & NF & 57.1 & 95.2 \\
\hline$\alpha$-Terpinene & St, MS & 121 & 95 & 75.0 & 85.7 & 71.4 & 52.4 \\
\hline |,4-Cineol & St, MS & 111 & 87 & 58.3 & 78.6 & 85.7 & 61.9 \\
\hline Limonene & St, MS & 68 & 84 & 33.3 & 57.1 & 57.1 & 28.6 \\
\hline 2-Heptanone & St, MS & 43 & 83 & 91.7 & 92.9 & 85.7 & 100.0 \\
\hline Heptanal & St, MS & 44 & 80 & 91.7 & NF & 14.3 & 95.2 \\
\hline 4-Methyl-2-heptanone & MS & 43 & 80 & 84.0 & NF & NF & 19.4 \\
\hline$\gamma$-Terpinene & St, MS & 93 & 89 & 58.3 & 92.9 & 100.0 & 57.1 \\
\hline$m$-Cymene ${ }^{b}$ & MS & 119 & 97 & 100.0 & 100.0 & 100.0 & 100.0 \\
\hline 2,2,6-Trimethyl-cyclohexanone & MS & 82 & 88 & 41.7 & 71.4 & 42.9 & 76.2 \\
\hline 2-Methoxythiophene & MS & 114 & 89 & 100.0 & 92.9 & 100.0 & 100.0 \\
\hline ।,2,4-Trimethylbenzene & MS & 105 & 90 & 83.3 & 85.7 & 100.0 & 95.2 \\
\hline Dimethyl trisulphide & St, MS & 126 & 91 & 83.3 & 100.0 & 100.0 & 100.0 \\
\hline 2-Methyl-5-(methylthio)furan & MS & 128 & 91 & 91.7 & 92.9 & 85.7 & 100.0 \\
\hline Nonanal & St, MS & 57 & 80 & 83.3 & 100.0 & 85.7 & 100.0 \\
\hline 1,2,3,4-Tetrahydro-1,5,7-trimethyl-naphthalene & MS & 159 & 90 & 66.7 & 71.4 & 85.7 & 81.0 \\
\hline p-Cymene & MS & 132 & 97 & 91.7 & 100.0 & 100.0 & 100.0 \\
\hline Linalyl oxide & MS & 59 & 82 & 83.3 & 92.9 & 100.0 & 90.5 \\
\hline Dihydrolinalool & MS & 73 & 84 & 16.7 & 28.6 & 57.1 & 76.2 \\
\hline Acetic acid ${ }^{b}$ & St, MS & 43 & 90 & 100.0 & 100.0 & 100.0 & 100.0 \\
\hline Furfural & St, MS & 96 & 91 & 100.0 & 92.9 & 100.0 & 100.0 \\
\hline 2,6-Dimethyl-7-octen-2-ol & St, MS & 59 & 90 & 91.7 & 100.0 & 100.0 & 100.0 \\
\hline I,2,3,4-Tetramethyl-benzene & MS & 119 & 80 & 50.0 & 28.6 & 57.1 & 66.7 \\
\hline Decanal & St, MS & 57 & 86 & 58.3 & 85.7 & 71.4 & 100.0 \\
\hline Bornylene & MS & 93 & 96 & 83.3 & 85.7 & 85.7 & 71.4 \\
\hline Vitispirane $\left.\right|^{b}$ & MS & 192 & 82 & 100.0 & 100.0 & 100.0 & 100.0 \\
\hline Vitispirane $\|^{\mathrm{b}}$ & MS & 192 & 85 & 100.0 & 100.0 & 100.0 & 100.0 \\
\hline 1,2,3,4-Tetrahydro- I, I,6-trimethyl-naphthalene & MS & 159 & 92 & 91.7 & 92.9 & 100.0 & 100.0 \\
\hline |-Octanol & St, MS & 56 & 90 & 91.7 & 85.7 & 71.4 & 100.0 \\
\hline Menthol & MS & 71 & 94 & 83.3 & 85.7 & 57.1 & 71.4 \\
\hline 2-Furanmethanol & St, MS & 98 & 80 & 83.3 & 78.6 & 57.1 & 85.7 \\
\hline 2-Methyl butanoic acid & St, MS & 74 & 83 & 91.7 & 92.9 & 100.0 & 81.0 \\
\hline Anisole & MS & 134 & 95 & 83.3 & 85.7 & 71.4 & 71.4 \\
\hline (+)-4-Carene & MS & 93 & 80 & 58.3 & 64.3 & 28.6 & 71.4 \\
\hline 2-Methyl-3-phenyl-2-propenal & St, MS & 145 & 80 & 91.7 & 85.7 & 100.0 & 100.0 \\
\hline 4-(I-Methylethyl)-I-cyclohexene-4-carboxaldehyde & MS & 109 & 85 & 41.7 & 42.9 & 57.1 & 19.0 \\
\hline 3-Carvomenthenone & MS & 82 & 80 & 33.3 & 42.9 & 28.6 & 33.3 \\
\hline D-Carvone & MS & 82 & 80 & 66.7 & 57.1 & 57.1 & 52.4 \\
\hline 1,2-Dihydro-1, 1,6-trimethyl-naphthalene b & St, MS & 157 & 97 & 100.0 & 100.0 & 100.0 & 100.0 \\
\hline I-(4-Methylphenyl)ethanone & MS & 119 & 94 & 33.3 & 28.6 & 14.3 & 14.3 \\
\hline I-Decanol & MS & 56 & 88 & 25.0 & 28.6 & 14.3 & 0.0 \\
\hline 4-(I-methylethyl)-benzaldehyde & MS & 133 & 84 & 58.3 & 14.3 & 57.1 & 9.5 \\
\hline 3,4-Dimethyl-benzaldehyde ${ }^{\mathrm{b}}$ & MS & 133 & 95 & 100.0 & 100.0 & 100.0 & 100.0 \\
\hline$\beta$-Damascenone ${ }^{\mathrm{b}}$ & St, MS & 69 & 86 & 100.0 & 100.0 & 100.0 & 100.0 \\
\hline p-Cymene-8-ol & MS & 43 & 90 & 66.7 & 85.7 & 100.0 & 100.0 \\
\hline 2-Methoxy-phenol & St, MS & 109 & 80 & 91.7 & 92.9 & 100.0 & 66.7 \\
\hline 2,7-Dimethyl-quinoline & St, MS & 157 & 85 & 83.3 & 85.7 & 85.7 & 85.7 \\
\hline Hexanoic acid & St, MS & 60 & 90 & 50.0 & 50.0 & 71.4 & 90.5 \\
\hline |-Dodecanol & MS & 55 & 89 & 8.3 & 7.1 & 14.3 & NF \\
\hline
\end{tabular}


Table 2 (Continued)

Frequency of occurrence (\%)

\begin{tabular}{|c|c|c|c|c|c|c|c|}
\hline Metabolites & $I D^{a}$ & $m / z$ & Match per cent & Colorectal & Leukaemia & Lymphoma & Control \\
\hline 2,6-Dimethyl-naphthalene & MS & 156 & 98 & 100.0 & 92.9 & 71.4 & 76.2 \\
\hline I-Ethyl-3,5-diisopropyl-benzene & MS & 175 & 88 & 100.0 & 100.0 & 100.0 & NF \\
\hline Phenolb & St, MS & 94 & 91 & 100.0 & 100.0 & 100.0 & 100.0 \\
\hline Octanoic acid & St, MS & 60 & 83 & 91.7 & 85.7 & 85.7 & 95.2 \\
\hline 4-Methyl-phenol & St, MS & 107 & 90 & 100.0 & 100.0 & 85.7 & 95.2 \\
\hline Hexadecanal & MS & 57 & 96 & 33.3 & NF & 28.6 & NF \\
\hline I,4,5-Trimethyl-naphthalene & MS & 155 & 86 & 66.7 & 78.6 & 71.4 & 57.1 \\
\hline$p$-Tert-butyl-phenol ${ }^{b}$ & MS & 135 & 96 & 100.0 & 100.0 & 100.0 & 100.0 \\
\hline 2,4-Bis( I,I-dimethylethyl)-phenol & MS & 191 & 95 & 100.0 & 92.9 & 85.7 & 100.0 \\
\hline Benzenecarboxilic acid & St, MS & 105 & 90 & 58.3 & 64.3 & 71.4 & 85.7 \\
\hline Indole & MS & 117 & 88 & 100.0 & 100.0 & 100.0 & 100.0 \\
\hline
\end{tabular}

Abbreviation: NF = not found. ${ }^{a}$ Metabolite identification using standard compound (st) or mass spectra of the NIST library search (MS). ${ }^{b}$ Metabolites identified in all 54 studied subject. 'Means that the metabolite was identified in all subjects of the corresponding group.

Table 3 Potential volatile marker metabolites found in the urinary volatile composition of the four groups by total significance of one-way ANOVA and LSD post-hoc test for multiple comparisons

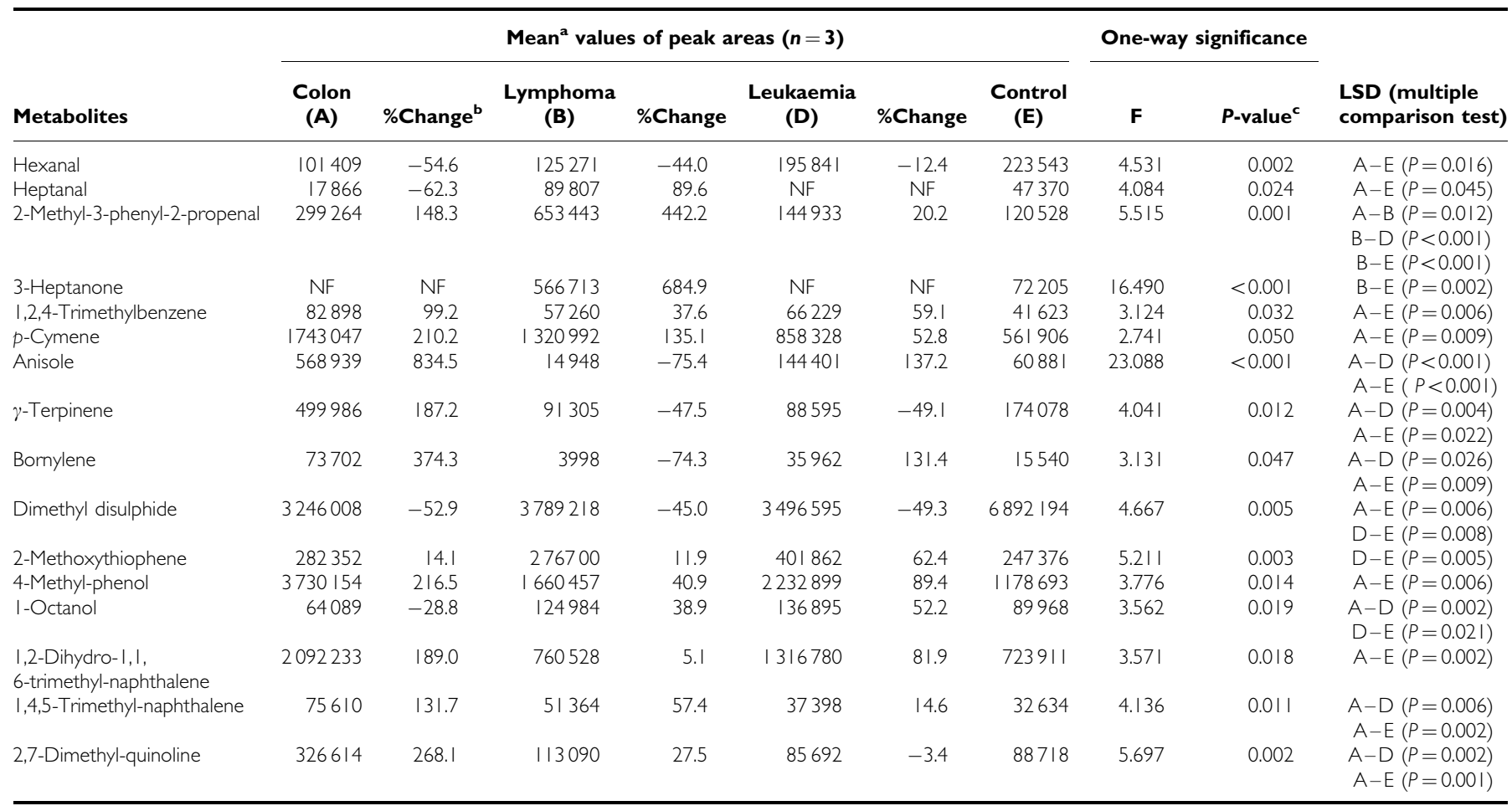

Abbreviations: ANOVA = analysis of variance; $\mathrm{LSD}=$ least significant difference; $\mathrm{NF}=$ not found; r.s.d. = relative standard deviation. ${ }^{\mathrm{a}}$ Average value from three replicates; r.s.d. lower than $20 \%$. ${ }^{b}$ Percentage change of cancer from normal, calculated from the arithmetic mean values of each group. Positive and negative percentages indicate higher levels of

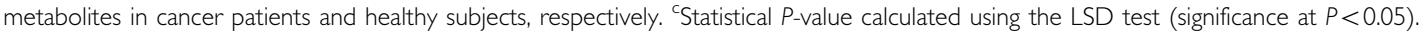

metabolomic profile of the oncological group (Figure $4 \mathrm{~A}$ ). The most abundant metabolites that belonged to these chemical classes were 4-heptanone, $p$-tert-butyl-phenol, 2,6-dimethyl-7-octen-2-ol and 1-ethyl-3,5-diisopropyl-benzene, respectively. The average areas of these metabolites indicated that when all cancer patients were combined into a single group, patients with cancer had higher levels of 3-methyl-3-phenyl-2-propenal, 3-heptanone, p-cymene, 2-methoxythiophene, phenol, 4-methyl-phenol and 1,2-dihydro-1, 1,6-trimethyl-naphthalene than the control group (Figure 4B). There was an increase in the amounts of all of these chemicals for all cancer patients when compared with the controls, except dimethyl disulphide, which was also a major compound that was identified to be present in the control group individuals.

The fragment ion $\mathrm{m} / \mathrm{z}$ values of the identified urinary metabolites with the highest abundance within each fragmentation pattern, the matching percentage of the NIST library and their 
frequency of occurrence in cancer patients and normal controls are listed in Table 2.

Among the 82 total metabolites identified, 21 were found in all (patients and controls) of the individuals who submitted to this study. As can be seen in Table 2, of the 82 identified metabolites, only 27 were common to colorectal cancer patients, whereas in the leukaemia and lymphoma cancer patients, the total number of common metabolites was 28 and 36, respectively. 3-Heptanone was only detected in half of the lymphoma patients, whereas 4-methyl2-heptanone was only identified in colorectal patients (84\%).

One-way ANOVA with $P_{\text {values }}<0.05$ was achieved for both groups (oncological and control) using SPSS, version 17.0. The positive rates of all 82 volatile metabolites were compared between the cancer group and the control group. Of the 82 identified metabolites, 16 were statistically significant (ANOVA, $P<0.05$ ) between the cancer patient and control groups, whereas the others did not show any significant differences. These volatile metabolites are summarised in Table 3.

Of the 16 metabolites of interest, it can be see that 3-heptanone, anisole, 2-methyl-3-phenyl-2-propenal, 2,7-dimethyl-quinoline and hexanal had the lowest $P$-values. If we looked more closely at the peak areas (Table 3), the urinary levels of 2-methyl-3phenyl-2-propenal, 3-heptanone and anisole that were found in healthy volunteers were much lower than those found in cancer patients. On the other hand, the urinary levels of hexanal and dimethyl disulphide were much higher in healthy persons than in cancer patients (Figure 5).

Metabolomic differences between the different types of investigated cancers were also detected (Figure 5). Such differences were manifested as higher levels of 2-methyl-3-phenyl-2-propenal and 3-heptanone and lower levels of aldehydes (hexanal and heptanal) in the lymphoma cancer patients in comparison with colorectal and leukaemia patients. Furthermore, urinary levels of $p$-cymene, anisole, $\gamma$-terpinene, bornylene, dimethyl disulphide, 4-methylphenol, 1,2-dihydro-1,1,6-trimethyl-naphthalene, 1,4,5-trimethylnaphthalene and 2,7-dimethyl-quinoline were higher in colorectal patients than in lymphoma and leukaemia patients. In addition, the increased content of sulphur-containing metabolites, namely 2-methoxythiophene and dimethyl disulphide, in leukaemia patients were statistically significant in comparison with normal controls (Figure 5).

The high inter-individual variability in urinary profiles and their complexity make any attempt at visual comparison of these spectra an unproductive task. Instead, multivariate analysis allowed the finding of consistent variation patterns within the data set. To study the principal sources of variation among the results, detect intrinsic clustering and possible outliers, and distinguish cancer patients from healthy individuals, exploratory PCA was applied to the GC-qMS peak areas that were obtained for the urinary volatile metabolites of both groups. This PCA is an unsupervised projection method that is used to visualise the data set and display similarities and differences. After preliminary statistical analysis, the PCA of the data showed that the variables described in Table 3 were enough to describe subsets with similar characteristics and relate to the health status of the subjects. Figure $6 \mathrm{~A}$ depicts the loadings of 16 variables on a plane defined by the first (PC1) and second (PC2) principal components.

Although this set of variables only explained $88.77 \%$ of the variability between the first two PCs, it was enough to deconvolute the set of cases in four subsets according to the health of the subjects and to cancer type. These results indicate a significant potential of early diagnosis of the studied cancer types using noninvasive urinary metabolomic analysis (Figure 6B).

The scores of the scatter plot demonstrate that PC2, which contained $25.13 \%$ of the total variability, split the samples into three different groups. The group located among the PC2-positive values corresponded to patients with lymphoma, whereas the second group, located among the negative values from PC1 and PC2, contained the leukaemia patients and normal controls. The third group, located among the PC1-positive and PC2-negative values, comprised the samples corresponding to the colorectal cancer subjects. These results demonstrate that the set of cases could be divided into four groups according to the clinical condition of the subjects.

The chemicals 3-heptanone (ZHept3), dimethyl disulphide (ZDisslf) and heptanal (ZHept) appear to play an important role in the healthy individuals group (control) and leukaemia patients.

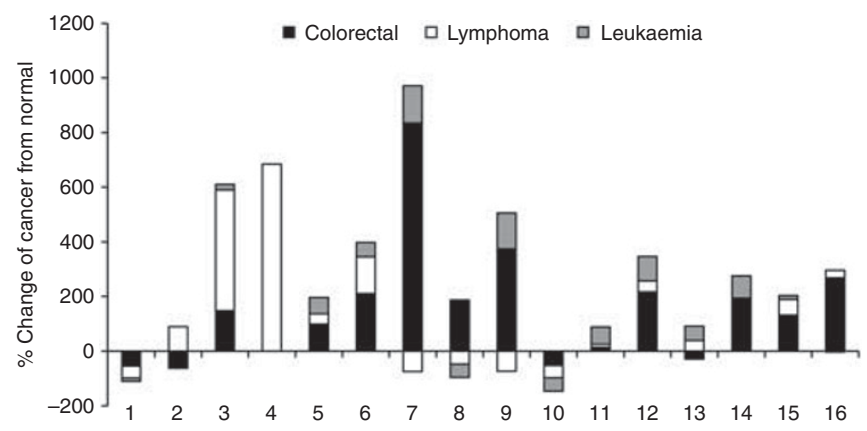

Figure 5 Percentage of change between cancer patients and normal subjects. Numbered bars correspond to: (1) hexanal; (2) heptanal; (3) 2 methyl-3-phenyl-2-propenal; (4) 3-heptanone; (5) I,2,4-trimethylbenzene; (6) p-cymene; (7) anisole; (8) $\gamma$-terpinene; (9) bornylene; (10) dimethyl disulphide; (I I) 2-methoxythiophene; ( 12) 4-methyl-phenol; ( 13) I-octanol; ( I4) I,2-dihydro- I, I,6-trimethyl-naphthalene; ( I 5) I,4,5-trimethyl-naphthalene; and (I6) 2,7-dimethyl-quinoline.
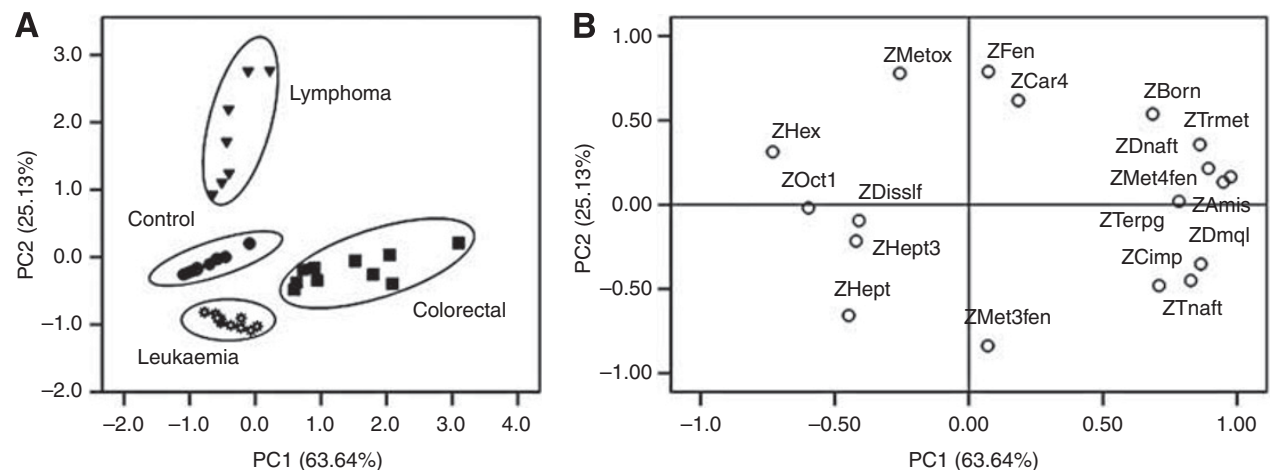

Figure 6 Separation of cancer patients and healthy individuals based on principal component analysis (PCA) scores scatter plot. (A) Loadings of variables on the PCI -PC2 plane (88.77\% of total variance). (B) Factor scores for PCA analysis showing the similarity groups for healthy persons (control group), and cancer patients as described by variables represented in Figure 4A. 
Colorectal cancer patients appear to be better characterised by 1,4,5-trimethyl-naphthalene (ZTnaft), 2,7-dimethyl-quinoline (ZDmql) and 2-methyl-3-phenyl-2-propenal (ZMet3fen). The chemicals 1-octanol (ZOct1), hexanal (ZHex) and 2-methoxythiophene (ZMetox) correlated well with the lymphoma subjects.

\section{CONCLUSIONS}

In this study, dHS-SPME/GC-qMS-based metabolomics was applied to investigate urinary cancer volatile metabolites as a useful tool to evaluate volatile metabolites for the early diagnosis of cancer. We found that dHS-SPME combined with GC-qMS was a simple, rapid, sensitive and solvent-free method that is highly suitable for this purpose. The potential advantages of urine analysis over other conventional medical tests include its noninvasive nature, low cost and safety. To achieve the highest recovery rate, the isolation procedure was optimised via the selection of an appropriate fibre, extraction temperature and extraction time using univariate optimisation design. A CAR/ PDMS fibre was found to be more sensitive for volatile metabolites than other coating phases. In all, 82 volatile metabolites were detected and identified in the control and oncological groups belonging to distinct chemical families, including aldehydes, ketones, terpenoids, acids, alcohols, benzene derivatives, furans and sulphur compounds, phenols, esters and naphthalene derivatives. Some of the metabolites were of endogenous origin and generated in the human body during normal metabolic processes, whereas some were inhaled with subsequent storage and later exhalation/excretion.

For the control group, the chemical families with the higher contributions to the urinary metabolomic profile were ketones and sulphur compounds. The major metabolites of these chemical families were 4-heptanone and methanethiol. Benzene derivatives, terpenes and volatile phenols were the chemical classes that exhibited higher contributions to the metabolomic patterns that were identified in the oncological group. Different VOM profiles for healthy people and cancer patients could be recognised by multivariate analysis, and significant diagnostic compounds could be established. Between the cancer group $(n=33)$ and the control group $(n=21)$, positive rates for 16 of the 82 detected compounds were found to be different with statistical significance $(P<0.05$, LSD test). There were definitive metabolomic differences between cancer patients and healthy individuals and between different types of cancer. The identification of volatile biomarkers in urine for disease diagnosis is an area of great promise, but it is based on limited previous human research. The data in this paper are consistent with the hypothesis that diagnostically useful volatile compounds are produced in patients with cancer and secreted into the urine, thus providing support for this diagnostic approach. The ability to easily collect and store urine samples will be a major advantage of this approach.

\section{ACKNOWLEDGEMENTS}

We thank the support of Portuguese Foundation for Science and Technology (FCT) through the MS Portuguese Networks (REDE/ 1508/RNEM/2005) and Pluriannual base funding (QUI-Madeira-674). We are grateful to the donors who kindly supplied the samples and to Haemato-Oncology Service of the Hospital Dr Nélio Mendonça (Funchal, Portugal) for the conditions to perform this study.

Supplementary Information accompanies the paper on British Journal of Cancer website (http://www.nature.com/bjc)

\section{REFERENCES}

Barrett JC, Anderson M (1993) Molecular mechanisms of carcinogenesis in humans and rodents. Mol Carcinogen 7: 1-13

Belda-Iniesta C, de Castro Carpeno J, Carrasco JA, Moreno V, Casado Saenz E, Feliu J, Sereno M, Garcia Rio F, Barriuso J, Gonzalez Baron M (2007) New screening method for lung cancer by detecting volatile organic compounds in breath. Clin Transl Oncol 9: 364-368

Carrola J, Rocha CM, Barros AS, Gil AM, Goodfellow BJ, Carreira IM, Bernardo J, Gomes A, Sousa V, Carvalho L, Duarte IF (2011) Metabolic signatures of lung cancer in biofluids: NMR-based metabonomics of urine. J Proteome Res 10: 221-230

Cassiday LT (2006) Weighing ribosomes with MS. Anal Chem 78: 7926

Chen J, Wang W, Lv S, Yin P, Zhao X, Lu X, Zhang F, Xu G (2009) Metabonomics study of liver cancer based on ultra performance liquid chromatography coupled to mass spectrometry with HILIC and RPLC separations. Anal Chim Acta 650: 3-9

Chen X, Xu F, Wang Y, Pan Y, Lu D, Wang P, Ying K, Chen E, Zhang W (2007) A study of the volatile organic compounds exhaled by lung cancer cells in vitro for breath diagnosis. Cancer 110: 835-844

Chikkaveeraiah BV, Bhirde A, Malhotra R, Patel V, Gutkind IS, Rusling JF (2009) Single-wall carbon nanotube forest arrays for immunoelectrochemical measurement of four protein biomarkers for prostate cancer. Anal Chem 81: 9129-9134

Deng C, Li N, Zhang X (2004a) Development of headspace solid-phase microextraction with on-fiber derivatization for determination of hexanal and heptanal in human blood. J Chromatogr B 813: 47-52

Deng C, Zhang X, Li N (2004b) Investigation of volatile biomarkers in lung cancer blood using solid-phase microextraction and capillary gas chromatography-mass spectrometry. J Chromatogr B 808: 269-277

Gaspar EM, Lopes JF (2009) Simple gas chromatographic method for furfural analysis. J Chromatogr A 1216: 2762-2767

Giardina M, Olesik SV (2003) Application of low-temperature glassy carbon-coated macrofibers for solid-phase microextraction analysis of simulated breath volatiles. Anal Chem 75: 1604-1614
Gramolini AO, Kislinger T, Alikhani-Koopaei R, Fong V, Thompson NJ, Isserlin R, Sharma P, Oudit GY, Trivieri MG, Fagan A, Kannan A, Higgins DG, Huedig H, Hess G, Arab S, Seidman JG, Seidman CE, Frey B, Perry M, Backx PH, Liu PP, MacLennan DH, Emili A (2008) Comparative proteomics profiling of a phospholamban mutant mouse model of dilated cardiomyopathy reveals progressive intracellular stress responses. Mol Cell Proteomics 7: 519-533

Greenberg AK, Lee MS (2007) Biomarkers for lung cancer: clinical uses. Curr Opin Pulm Med 13: 249-255

Henneges C, Bullinger D, Fux R, Friese N, Seeger H, Neubauer H, Laufer S, Gleiter CH, Schwab M, Zell A, Kammerer B (2009) Prediction of breast cancer by profiling of urinary RNA metabolites using support vector machine-based feature selection. BMC Cancer 9: 104

Infante PF, Schuman LD, Dement J, Huff J (1994) Fibrous glass and cancer. Am I Ind Med 26: 559-584

Jemal A, Siegel R, Xu J, Ward E (2010) Cancer statistics, 2010. CA Cancer J Clin 60: $277-300$

Jentzmik F, Stephan C, Miller K, Schrader M, Erbersdobler A, Kristiansen G, Lein M, Jung K (2010) Sarcosine in urine after digital rectal examination fails as a marker in prostate cancer detection and identification of aggressive tumours. Eur Urol 58: 12-18; discussion $20-1$

Jiang Y, Ma Y (2009) A fast capillary electrophoresis method for separation and quantification of modified nucleosides in urinary samples. Anal Chem 81: $6474-6480$

Lauridsen M, Hansen SH, Jaroszewski JW, Cornett C (2007) Human urine as test material in ${ }^{1} \mathrm{H}$ NMR-based metabonomics: recommendations for sample preparation and storage. Anal Chem 79: $1181-1186$

Ma YL, Qin HL, Liu WJ, Peng JY, Huang L, Zhao XP, Cheng YY (2009) Ultra-high performance liquid chromatography-mass spectrometry for the metabolomic analysis of urine in colorectal cancer. Dig Dis Sci 54: $2655-2662$

Matsumura K, Opiekun M, Oka H, Vachani A, Albelda SM, Yamazaki K, Beauchamp GK (2010) Urinary volatile compounds as biomarkers for 
lung cancer: a proof of principle study using odor signatures in mouse models of lung cancer. PLoS One 5: e8819

Mazzone PJ (2008) Analysis of volatile organic compounds in the exhaled breath for the diagnosis of lung cancer. $J$ Thorac Oncol 3: $774-780$

McCulloch M, Jezierski T, Broffman M, Hubbard A, Turner K, Janecki T (2006) Diagnostic accuracy of canine scent detection in early- and latestage lung and breast cancers. Integr Cancer Ther 5: 30-39

Minamoto T, Mai M, Ronai Z (2000) K-ras mutation: early detection in molecular diagnosis and risk assessment of colorectal, pancreas, and lung cancers-a review. Cancer Detect Prev 24: $1-12$

Musteata FM, Pawliszyn J (2007) Bioanalytical applications of solid-phase microextraction. Trends Anal Chem 26: $36-45$

Oliveira PA, Colaco A, Chaves R, Guedes-Pinto H, De-La-Cruz PL, Lopes C (2007) Chemical carcinogenesis. An Acad Bras Cienc 79: 593-616

Ouyang G, Chen Y, Setkova L, Pawliszyn J (2005) Calibration of solid-phase micro-extraction for quantitative analysis by gas chromatography. J Chromatogr A 1097: 9-16

Phillips M, Cataneo RN, Ditkoff BA, Fisher P, Greenberg J, Gunawardena R, Kwon CS, Rahbari-Oskoui F, Wong C (2003) Volatile markers of breast cancer in the breath. Breast J 9: 184-191

Phillips M, Cataneo RN, Ditkoff BA, Fisher P, Greenberg J, Gunawardena R, Kwon CS, Tietje O, Wong C (2006) Prediction of breast cancer using volatile biomarkers in the breath. Breast Cancer Res Treat 99: 19-21

Prado C, Marin P, Periago JF (2003) Application of solid-phase microextraction and gas chromatography-mass spectrometry to the determination of volatile organic compounds in end-exhaled breath samples. J Chromatogr A 1011: 125-134
Qiu Y, Cai G, Su M, Chen T, Liu Y, Xu Y, Ni Y, Zhao A, Cai S, Xu LX, Jia W (2010) Urinary metabonomic study on colorectal cancer. J Proteome Res 9: $1627-1634$

Song G, Qin T, Liu H, Xu GB, Pan YY, Xiong FX, Gu KS, Sun GP, Chen ZD (2010) Quantitative breath analysis of volatile organic compounds of lung cancer patients. Lung Cancer 67: 227-231

Sreekumar A, Poisson LM, Rajendiran TM, Khan AP, Cao Q, Yu J, Laxman B, Mehra R, Lonigro RJ, Li Y, Nyati MK, Ahsan A, Kalyana-Sundaram S, Han B, Cao X, Byun J, Omenn GS, Ghosh D, Pennathur S, Alexander DC, Berger A, Shuster JR, Wei JT, Varambally S, Beecher C, Chinnaiyan AM (2009) Metabolomic profiles delineate potential role for sarcosine in prostate cancer progression. Nature 457: 910 -914

Ullah MF, Aatif M (2009) The footprints of cancer development: cancer biomarkers. Cancer Treat Rev 35: 193-200

Walker V, Mills GA (2001) Urine 4-heptanone: a beta-oxidation product of 2-ethylhexanoic acid from plasticisers. Clin Chim Acta 306: 51-61

Weisburger JH, Williams GM (2000) The distinction between genotoxic and epigenetic carcinogens and implication for cancer risk. Toxicol Sci 57: 4-5

Xue R, Dong L, Zhang S, Deng C, Liu T, Wang J, Shen X (2008) Investigation of volatile biomarkers in liver cancer blood using solidphase microextraction and gas chromatography/mass spectrometry. Rapid Commun Mass Spectrom 22: 1181-1186

Yu Q, Huang R, Li L, Lin L, Hang W, He J, Huang B (2009) Applicability of standardless semiquantitative analysis of solids by high-irradiance laser ionization orthogonal time-of-fight mass spectrometry. Anal Chem 81: $4343-4348$

Yung TCH (2010) Is that lung cancer I smell in your breath? Univ Toronto Med J 87: $122-123$

This work is published under the standard license to publish agreement. After 12 months the work will become freely available and the license terms will switch to a Creative Commons Attribution-NonCommercial-Share Alike 3.0 Unported License. 